CREAT. MATH. INFORM.

Volume 29 (2020), No. 2,

Pages 153 - 160
Online version at https : //creative-mathematics. cunbm . utcluj. ro/

Print Edition: ISSN 1584 - 286X; Online Edition: ISSN 1843 - 441X

DOI: https://doi.org/10.37193/CMI.2020.02.06

\title{
A relation between the Brocard and Miquel Angles
}

\author{
SÁNDOR NAGYDOBAI KISS
}

ABSTRACT. Let $X Y Z$ be an inscribed triangle in the reference triangle $A B C(X \in B C, Y \in C A, Z \in A B)$ given by its absolute barycentric coordinates: $X=(0,1-\alpha, \alpha), Y=(\beta, 0,1-\beta), Z=(1-\gamma, \gamma, 0)$, where $\alpha, \beta, \gamma$ are arbitrary real numbers. In this paper we deduce the following simple and beautiful formula between the Brocard and Miquel angles: $S_{\omega}=S_{\theta}+a^{2} \alpha+b^{2} \beta+c^{2} \gamma$, where $\omega$ is the Brocard and $\theta$ the Miquel angle of $A B C$.

\section{Preliminaries}

In this paper we will use the barycentric coordinates [1] and the Conway triangle notations [2]. Given a reference triangle $A B C$ whose sides are $a, b$ and $c$ and whose corresponding internal angles $A, B$ and $C$, then the Conway triangle notations are

$$
S=2 \times \text { Area } A B C=b c \sin A=c a \sin B=a b \sin C \text { and } S_{\varphi}=S \cot \varphi,
$$

in particular $S_{A}=b c \cos A, S_{B}=c a \cos B, S_{C}=a b \cos C$. We will use also some important conditioned trigonometric identities:

$$
\begin{gathered}
b^{2} c^{2}-S_{A}^{2}=c^{2} a^{2}-S_{B}^{2}=a^{2} b^{2}-S_{C}^{2}=S^{2} ; \\
a^{2} S_{A}+S_{B C}=b^{2} S_{B}+S_{C A}=c^{2} S_{C}+S_{A B}=S^{2} ; \\
S_{B C}+S_{C A}+S_{A B}=S^{2} ; \\
a^{2} S_{A}+b^{2} S_{B}+c^{2} S_{C}=2 S^{2} ; \\
S_{\omega}=\frac{a^{2}+b^{2}+c^{2}}{2}=S_{A}+S_{B}+S_{C}=a^{2}+S_{A}=b^{2}+S_{B}=c^{2}+S_{C} ; \\
b^{2} c^{2}+c^{2} a^{2}+a^{2} b^{2}-S_{\omega}^{2}=S^{2} .
\end{gathered}
$$

We draw a circle through points $B$ and $C$, tangent to side $C A$, another circle through points $C$ and $A$, tangent to side $A B$ and a circle through points $A$ and $B$, tangent to side $B C$ of the triangle $A B C$ [3]. These three circles have a common point $(\Omega)$, the first Brocard point of triangle $A B C$ (Figure 1). Moreover, $\measuredangle \Omega A B=\measuredangle \Omega B C=\measuredangle \Omega C A=\omega$, and this angle $\omega$ is called the Brocard angle of the triangle $A B C$. The Brocard angle has the property that

$$
\cot \omega=\cot A+\cot B+\cot C=\frac{a^{2}+b^{2}+c^{2}}{2 S} .
$$

Similarly we can construct the second Brocard point $\left(\Omega^{\prime}\right)$.

Received: 08.03.2020. In revised form: 13.09.2020. Accepted: 20.09.2020

2010 Mathematics Subject Classification. 51N20.

Key words and phrases. Miquel angle, Brocard angle, barycentric coordinates. 


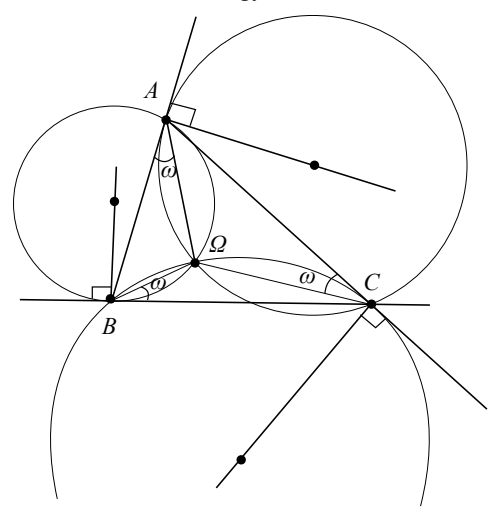

Figure 1.

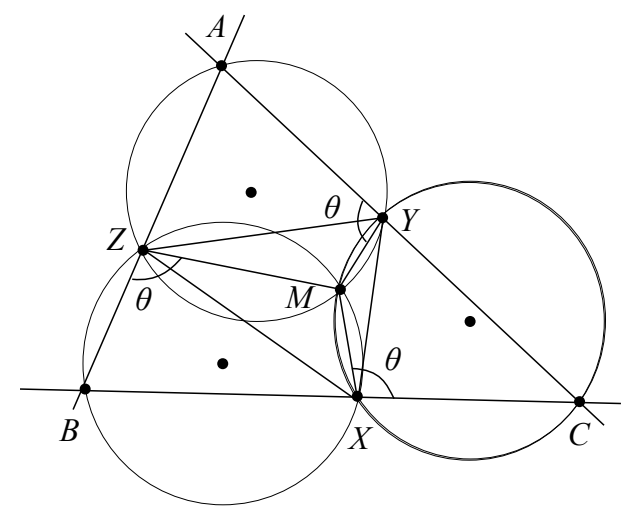

Figure 2.

The three circumcircles to triangles $A Y Z, B Z X$ and $C X Y$ intersect in a single point $M$, called the Miquel's point of the triangle $A B C$ [4]. In addition $\measuredangle A Y M=\measuredangle B Z M=$ $\measuredangle C X M=\theta$, and this angle $\theta$ is called the Miquel angle of $A B C$ (Figure 2).

\section{EQUATIONS OF CIRCUMCIRCLES OF THE RESIDUAL TRIANGLES}

Firstly we will determine the barycentric coordinates of the circumcenters $O_{a}, O_{b}, O_{c}$ of the residual triangles $A Y Z, B Z X, C X Y$ respectively. Denote with $L(K, P Q)$ the perpendicular from the point $K$ to the line $P Q$. Consequently $O_{a}=L\left(Y_{a}, A C\right) \cap L\left(Z_{a}, A B\right)$, where $Y_{a}$ and $Z_{a}$ are the midpoints of segments $A Y$ and $A Z$. So $2 Y_{a}=(1+\beta, 0,1-\beta)$ and $2 Z_{a}=(2-\gamma, \gamma, 0)$ (Figure 3).

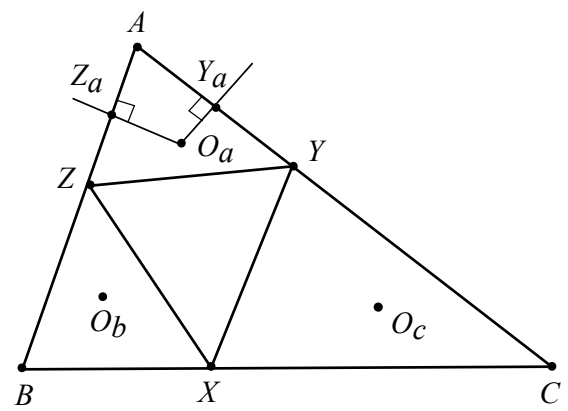

Figure 3. 
The equations of the perpendiculars $L\left(Y_{a}, A C\right)$ and $L\left(Z_{a}, A B\right)$ are

$$
\begin{aligned}
& \left|\begin{array}{ccc}
x & y & z \\
1+\beta & 0 & 1-\beta \\
S_{C} & -b^{2} & S_{A}
\end{array}\right|=0 \Leftrightarrow b^{2}(1-\beta) x+\left(S_{C}-S_{A}-b^{2} \beta\right) y-b^{2}(1+\beta) z=0, \\
& \left|\begin{array}{ccc}
x & y & z \\
2-\gamma & \gamma & 0 \\
S_{B} & S_{A} & -c^{2}
\end{array}\right|=0 \Leftrightarrow-c^{2} \gamma x+c^{2}(2-\gamma) y+\left(2 S_{A}-c^{2} \gamma\right) z=0 .
\end{aligned}
$$

With the Cramer rule from these equations we can obtain the coordinates of $O_{a}$ :

$$
\begin{aligned}
\delta & =\left|\begin{array}{cc}
b^{2}(1-\beta) & S_{C}-S_{A}-b^{2} \beta \\
-c^{2} \gamma & c^{2}(2-\gamma)
\end{array}\right|=b^{2} c^{2}(1-\beta)-c^{2} S_{A} \gamma ; \\
\delta_{x} & =\left|\begin{array}{cc}
b^{2}(1+\beta) & S_{C}-S_{A}-b^{2} \beta \\
-\left(2 S_{A}-c^{2} \gamma\right) & c^{2}(2-\gamma)
\end{array}\right| z=\left[2 S^{2}-b^{2} S_{B}(1-\beta)-c^{2} S_{C} \gamma\right] z ; \\
\delta_{y} & =\left|\begin{array}{cc}
b^{2}(1-\beta) & b^{2}(1+\beta) \\
-c^{2} \gamma & -\left(2 S_{A}-c^{2} \gamma\right)
\end{array}\right| z=\left[-b^{2} S_{A}(1-\beta)+b^{2} c^{2} \gamma\right] z .
\end{aligned}
$$

Consequently the coordinates of $O_{a}$ are

$$
O_{a}=\left(2 S^{2}-b^{2} S_{B}(1-\beta)-c^{2} S_{C} \gamma:-b^{2} S_{A}(1-\beta)+b^{2} c^{2} \gamma: b^{2} c^{2}(1-\beta)-c^{2} S_{A} \gamma\right) .
$$

The sum of these coordinates is $2 S^{2}$, so the absolute barycentric coordinates of $O_{a}$ are

$$
O_{a}=\left(1-\frac{b^{2} S_{B}(1-\beta)+c^{2} S_{C} \gamma}{2 S^{2}}, \frac{-b^{2} S_{A}(1-\beta)+b^{2} c^{2} \gamma}{2 S^{2}}, \frac{b^{2} c^{2}(1-\beta)-c^{2} S_{A} \gamma}{2 S^{2}}\right) .
$$

Similarly we obtain the absolute barycentric coordinates of $O_{b}$ and $O_{c}$ :

$$
\begin{aligned}
O_{b} & =\left(\frac{c^{2} a^{2}(1-\gamma)-a^{2} S_{B} \alpha}{2 S^{2}}, 1-\frac{c^{2} S_{C}(1-\gamma)+a^{2} S_{A} \alpha}{2 S^{2}}, \frac{-c^{2} S_{B}(1-\gamma)+c^{2} a^{2} \alpha}{2 S^{2}}\right) . \\
O_{c} & =\left(\frac{-a^{2} S_{C}(1-\alpha)+a^{2} b^{2} \beta}{2 S^{2}}, \frac{a^{2} b^{2}(1-\alpha)-b^{2} S_{C} \beta}{2 S^{2}}, 1-\frac{a^{2} S_{A}(1-\alpha)+b^{2} S_{B} \beta}{2 S^{2}}\right) .
\end{aligned}
$$

Denote with $O_{a}\left(R_{a}\right), O_{b}\left(R_{b}\right), O_{c}\left(R_{c}\right)$ the circumcircles of the residual triangles $A Y Z$, $B Z X, C X Y$ respectively, where $R_{a}, R_{b}, R_{c}$ are the radii of these circles.

\section{Proposition 2.1.}
(a) $R_{a}=\frac{b c}{2 S} \sqrt{b^{2}(1-\beta)^{2}-2(1-\beta) \gamma S_{A}+c^{2} \gamma^{2}}$,
(b) $R_{b}=\frac{c a}{2 S} \sqrt{c^{2}(1-\gamma)^{2}-2(1-\gamma) \alpha S_{B}+a^{2} \alpha^{2}}$,
(c) $R_{c}=\frac{a b}{2 S} \sqrt{a^{2}(1-\alpha)^{2}-2(1-\alpha) \beta S_{C}+b^{2} \beta^{2}}$.

Proof. We have:

$$
\begin{gathered}
R_{a}^{2}=A O_{a}^{2}=\left(\frac{b^{2} S_{B}(1-\beta)+c^{2} S_{C} \gamma}{2 S^{2}}\right)^{2} S_{A}+\left(\frac{-b^{2} S_{A}(1-\beta)+b^{2} c^{2} \gamma}{2 S^{2}}\right)^{2} S_{B} \\
+\left(\frac{b^{2} c^{2}(1-\beta)-c^{2} S_{A} \gamma}{2 S^{2}}\right)^{2} S_{C}=\frac{1}{4 S^{4}}\left[b^{4}\left(S_{B}^{2} S_{A}+S_{A}^{2} S_{B}+c^{4} S_{C}\right)(1-\beta)^{2}\right. \\
\left.+2 b^{2} c^{2} S_{A}\left(S_{B} S_{C}-b^{2} S_{B}-c^{2} S_{C}\right)(1-\beta) \gamma+c^{4}\left(S_{C}^{2} S_{A}+S_{A}^{2} S_{C}+b^{4} S_{B}\right) \gamma^{2}\right] \\
=\frac{b^{2} c^{2}}{4 S^{2}}\left[b^{2}(1-\beta)^{2}-2(1-\beta) \gamma S_{A}+c^{2} \gamma^{2}\right] .
\end{gathered}
$$


We will introduce the following notations:

$$
\begin{aligned}
\lambda & =\lambda(\alpha, \beta, \gamma)=a^{2} \alpha+b^{2} \beta+c^{2} \gamma, \\
\lambda^{\prime} & =\lambda(1-\alpha, 1-\beta, 1-\gamma)=a^{2}(1-\alpha)+b^{2}(1-\beta)+c^{2}(1-\gamma), \\
E & =E(\alpha, \beta, \gamma)=S_{\omega}-\lambda=\frac{1}{2}\left[a^{2}(1-2 \alpha)+b^{2}(1-2 \beta)+c^{2}(1-2 \gamma)\right] .
\end{aligned}
$$

Consequently $\lambda^{\prime}=2 S_{\omega}-\lambda$ i.e. $\lambda+\lambda^{\prime}=2 S_{\omega}=a^{2}+b^{2}+c^{2}$ or $S_{\omega}-\lambda=\lambda^{\prime}-S_{\omega}$.

\section{Proposition 2.2.}

(a) $O_{b} O_{c}=\frac{a}{2 S} \sqrt{E^{2}+S^{2}}=\frac{a}{2 S} \sqrt{\left(a^{2} \alpha+b^{2} \beta+c^{2} \gamma-S_{\omega}\right)^{2}+S^{2}}$,

(b) $O_{c} O_{a}=\frac{b}{2 S} \sqrt{E^{2}+S^{2}}=\frac{b}{2 S} \sqrt{\left(a^{2} \alpha+b^{2} \beta+c^{2} \gamma-S_{\omega}\right)^{2}+S^{2}}$,

(c) $O_{a} O_{b}=\frac{c}{2 S} \sqrt{E^{2}+S^{2}}=\frac{c}{2 S} \sqrt{\left(a^{2} \alpha+b^{2} \beta+c^{2} \gamma-S_{\omega}\right)^{2}+S^{2}}$.

Proof. We calculate the differences of coordinates of the points $O_{b}$ and $O_{c}$ :

$$
\begin{aligned}
x_{O_{b}}-x_{O_{c}} & =\frac{1}{2 S^{2}}\left[c^{2} a^{2}(1-\gamma)-a^{2} S_{B} \alpha+a^{2} S_{C}(1-\alpha)-a^{2} b^{2} \beta\right] \\
& =\frac{a^{2}}{2 S^{2}}\left(c^{2}+S_{C}-a^{2} \alpha-b^{2} \beta-c^{2} \gamma\right)=\frac{a^{2}\left(S_{\omega}-\lambda\right)}{2 S^{2}}, \\
y_{O_{b}}-y_{O_{c}} & =\frac{1}{2 S^{2}}\left[2 S^{2}-c^{2} S_{C}(1-\lambda)-a^{2} S_{A} \alpha-a^{2} b^{2}(1-\alpha)+b^{2} S_{C} \beta\right] \\
& =\frac{1}{2 S^{2}}\left[S_{C}\left(a^{2} \alpha+b^{2} \beta+c^{2} \gamma-S_{\omega}\right)+S^{2}\right]=\frac{S_{C}\left(\lambda-S_{\omega}\right)+S^{2}}{2 S^{2}}, \\
z_{O_{b}}-z_{O_{c}} & =\frac{1}{2 S^{2}}\left[-c^{2} S_{B}(1-\gamma)+c^{2} a^{2} \alpha-2 S^{2}+a^{2} S_{A}(1-\alpha)+b^{2} S_{B} \beta\right] \\
& =\frac{1}{2 S^{2}}\left[S_{B}\left(a^{2} \alpha+b^{2} \beta+c^{2} \gamma-S_{\omega}\right)-S^{2}\right]=\frac{S_{B}\left(\lambda-S_{\omega}\right)-S^{2}}{2 S^{2}} .
\end{aligned}
$$

The length of segment $O_{b} O_{c}$ is

$$
\begin{aligned}
& \left(O_{b} O_{c}\right)^{2}=\left(\frac{a^{2}\left(S_{\omega}-\lambda\right)}{2 S^{2}}\right)^{2} S_{A}+\left(\frac{S_{C}\left(\lambda-S_{\omega}\right)+S^{2}}{2 S^{2}}\right)^{2} S_{B}+\left(\frac{S_{B}\left(\lambda-S_{\omega}\right)-S^{2}}{2 S^{2}}\right)^{2} S_{C} \\
& =\frac{1}{4 S^{4}}\left[\left(a^{4} S_{A}+S_{B} S_{C}^{2}+S_{B}^{2} S_{C}\right)\left(\lambda-S_{\omega}\right)^{2}+\left(S_{B}+S_{C}\right) S^{4}\right]=\frac{a^{2}\left(\left(\lambda-S_{\omega}\right)^{2}+S^{2}\right)}{4 S^{2}} .
\end{aligned}
$$

Proposition 2.3. The equations of circumcircles of triangles $A Y Z, B Z X, C X Y$ are:

$$
\begin{aligned}
& O_{a}\left(R_{a}\right): S_{A} x^{2}+S_{B} y^{2}+S_{C} z^{2}-(x+y+z)\left[S_{A} x-\left(S_{A}-c^{2} \gamma\right) y+\left(S_{C}-b^{2} \beta\right) z\right]=0, \\
& O_{b}\left(R_{b}\right): S_{A} x^{2}+S_{B} y^{2}+S_{C} z^{2}-(x+y+z)\left[\left(S_{A}-c^{2} \gamma\right) x+S_{B} y-\left(S_{B}-a^{2} \alpha\right) z\right]=0, \\
& O_{c}\left(R_{c}\right): S_{A} x^{2}+S_{B} y^{2}+S_{C} z^{2}-(x+y+z)\left[-\left(S_{C}-b^{2} \beta\right) x+\left(S_{B}-a^{2} \alpha\right) y+S_{C} z\right]=0 .
\end{aligned}
$$


Proof. The equation of the circle $O_{a}\left(R_{a}\right)$ is $P O_{a}^{2}=R_{a}^{2} \Leftrightarrow P O_{a}^{2}=A O_{a}^{2}$, where $P=$ $\left(\frac{x}{\mu}, \frac{y}{\mu}, \frac{z}{\mu}\right), \mu=x+y+z \neq 0$. So

$$
\begin{gathered}
\left(\frac{x}{\mu}-x_{O_{a}}\right)^{2} S_{A}+\left(\frac{y}{\mu}-y_{O_{a}}\right)^{2} S_{B}+\left(\frac{z}{\mu}-z_{O_{a}}\right)^{2} S_{C} \\
=\left(1-x_{O_{a}}\right)^{2} S_{A}+y_{O_{a}}^{2} S_{B}+z_{O_{a}}^{2} S_{C} \Leftrightarrow S_{A} x^{2}+S_{B} y^{2}+S_{C} z^{2}- \\
-2 \mu\left(S_{A} x_{O_{a}} x+S_{B} y_{O_{a}} y+S_{C} z_{O_{a}} z\right)-\mu^{2}\left(1-2 x_{O_{a}}\right) S_{A}=0 \\
S_{A} x^{2}+S_{B} y^{2}+S_{C} z^{2}-\mu\left[S_{A} x+\left(S_{A}-2 S_{A} x_{O_{a}}+2 S_{B} y_{O_{a}}\right) y+\right. \\
\left.+\left(S_{A}-2 S_{A} x_{O_{a}}+2 S_{C} z_{O_{a}}\right)\right]=0 \\
\Leftrightarrow S_{A} x^{2}+S_{B} y^{2}+S_{C} z^{2}-(x+y+z)\left[S_{A} x-\left(S_{A}-c^{2} \gamma\right) y+\left(S_{C}-b^{2} \beta\right) z\right]=0 .
\end{gathered}
$$

\section{BARYCENTRIC COORDINATES OF THE MIQUEL POINT}

Denote with $L_{b c}, L_{c a}, L_{a b}$ the radical axes of the pairs of circles $\left(O_{b}\left(R_{b}\right), O_{c}\left(R_{c}\right)\right), O_{c}\left(R_{c}\right)$, $\left.O_{a}\left(R_{a}\right)\right),\left(O_{a}\left(R_{a}\right), O_{b}\left(R_{b}\right)\right)$ respectively.

Proposition 3.4. The equations of the radical axes $L_{b c}, L_{c a}, L_{a b}$ are

$$
\begin{aligned}
& L_{b c}:\left[b^{2}(1-\beta)-c^{2} \gamma\right] x+a^{2} \alpha y-a^{2}(1-\alpha) z=0, \\
& L_{c a}:-b^{2}(1-\beta) x+\left[c^{2}(1-\gamma)-a^{2} \alpha\right] y+b^{2} \beta z=0, \\
& L_{a b}: c^{2} \gamma x-c^{2}(1-\gamma) y+\left[a^{2}(1-\alpha)-b^{2} \beta\right] z=0 .
\end{aligned}
$$

Proof. We obtain the equation of the radical axis $L_{b c}$ as the difference of the equations of circles $O_{b}\left(R_{b}\right)$ and $O_{c}\left(R_{c}\right)$, i.e.

$$
\begin{gathered}
\left(S_{A}-c^{2} \gamma\right) x+S_{B} y-\left(S_{B}-a^{2} \alpha\right) z-\left[-\left(S_{C}-b^{2} \beta\right) x+\left(S_{B}-a^{2} \alpha\right) y+S_{C} z\right]=0 \\
\Leftrightarrow\left[b^{2}(1-\beta)-c^{2} \gamma\right] x+a^{2} \alpha y-a^{2}(1-\alpha) z=0 .
\end{gathered}
$$

Proposition 3.5. The radical axes $L_{b c}, L_{c a}, L_{a b}$ are concurrent.

Proof. In the equations of the radical axes $L_{b c}, L_{c a}, L_{a b}$ the sums of the coefficients of $x, y$ respectively $\mathrm{z}$ are zero.

Let $M$ be the point of concurrence of the radical axes $L_{b c}, L_{c a}, L_{a b}$. This point is called the radical center of circles $O_{a}\left(R_{a}\right), O_{b}\left(R_{b}\right), O_{c}\left(R_{c}\right)$ or the Miquel point determined by the inscribed triangle $X Y Z$ (Figure 4 ):

$$
M=L_{b c} \cap L_{c a} \cap L_{a b}=O_{a}\left(R_{a}\right) \cap O_{b}\left(R_{b}\right) \cap O_{c}\left(R_{c}\right) .
$$

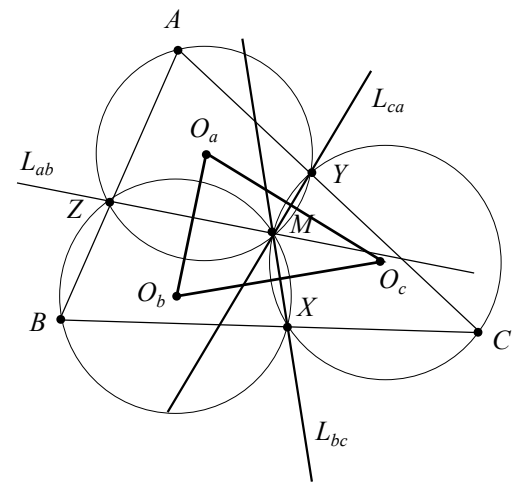

Figure 4 . 
From the equations of radical axis $L_{b c}$ and $L_{c a}$, applied the Cramer rule we can determine the barycentric coordinates of Miquel point:

$$
\begin{aligned}
& \delta=\left|\begin{array}{cc}
b^{2}(1-\beta)-c^{2} \gamma & a^{2} \alpha \\
-b^{2}(1-\beta) & c^{2}(1-\gamma)-a^{2} \alpha
\end{array}\right|=\left|\begin{array}{cc}
b^{2}(1-\beta)-c^{2} \gamma & a^{2} \alpha \\
-c^{2} \gamma & c^{2}(1-\gamma)
\end{array}\right| \\
& =c^{2}\left[a^{2} \alpha+(1-\gamma)\left(b^{2}-a^{2} \alpha-b^{2} \beta-c^{2} \gamma\right)\right]=c^{2}\left[\left(b^{2}-\lambda\right)(1-\gamma)+a^{2} \alpha\right] \text {; } \\
& \delta_{x}=\left|\begin{array}{cc}
a^{2}(1-\alpha) & a^{2} \alpha \\
-b^{2} \beta & c^{2}(1-\gamma)-a^{2} \alpha
\end{array}\right| z=a^{2}\left[\left(c^{2}-\lambda\right)(1-\alpha)+b^{2} \beta\right] z ; \\
& \delta_{y}=\left|\begin{array}{cc}
b^{2}(1-\beta)-c^{2} \gamma & a^{2}(1-\alpha) \\
-b^{2}(1-\beta) & -b^{2} \beta
\end{array}\right| z=b^{2}\left[\left(a^{2}-\lambda\right)(1-\beta)+c^{2} \gamma\right] z .
\end{aligned}
$$

Therefore

$$
M=\left(a^{2}\left[\left(c^{2}-\lambda\right)(1-\alpha)+b^{2} \beta\right]: b^{2}\left[\left(a^{2}-\lambda\right)(1-\beta)+c^{2} \gamma\right]: c^{2}\left[\left(b^{2}-\lambda\right)(1-\gamma)+a^{2} \alpha\right]\right) .
$$

We denote with $\mu_{P}$ the sum of coordinates of an arbitrary point $P$.

Therefore:

$$
\begin{aligned}
\mu_{M} & =b^{2} c^{2}+c^{2} a^{2}+a^{2} b^{2}-\lambda\left[a^{2}(1-\alpha)+b^{2}(1-\beta)+c^{2}(1-\gamma)\right] \\
& =b^{2} c^{2}+c^{2} a^{2}+a^{2} b^{2}-\lambda \lambda^{\prime}=b^{2} c^{2}+c^{2} a^{2}+a^{2} b^{2}+\lambda\left(\lambda-2 S_{\omega}\right) \\
& =b^{2} c^{2}+c^{2} a^{2}+a^{2} b^{2}+\left(\lambda-S_{\omega}\right)^{2}-S_{\omega}^{2}=\left(\lambda-S_{\omega}\right)^{2}+S^{2}=\left(S_{\omega}-\lambda^{\prime}\right)^{2}+S^{2} .
\end{aligned}
$$

Let $x_{M}, y_{M}, z_{M}$ be the absolute barycentric coordinates of $M$ :

$$
\begin{aligned}
& x_{M}=\frac{a^{2}\left[\left(c^{2}-\lambda\right)(1-\alpha)+b^{2} \beta\right]}{\left(\lambda-S_{\omega}\right)^{2}+S^{2}}=\frac{a^{2}\left[c^{2}(1-\gamma)+\left(b^{2}-\lambda^{\prime}\right) \alpha\right]}{\left(\lambda-S_{\omega}\right)^{2}+S^{2}}, \\
& y_{M}=\frac{b^{2}\left[\left(a^{2}-\lambda\right)(1-\beta)+c^{2} \gamma\right]}{\left(\lambda-S_{\omega}\right)^{2}+S^{2}}=\frac{b^{2}\left[a^{2}(1-\alpha)+\left(c^{2}-\lambda^{\prime}\right) \beta\right]}{\left(\lambda-S_{\omega}\right)^{2}+S^{2}}, \\
& z_{M}=\frac{c^{2}\left[\left(b^{2}-\lambda\right)(1-\gamma)+a^{2} \alpha\right]}{\left(\lambda-S_{\omega}\right)^{2}+S^{2}}=\frac{c^{2}\left[b^{2}(1-\beta)+\left(a^{2}-\lambda^{\prime}\right) \gamma\right]}{\left(\lambda-S_{\omega}\right)^{2}+S^{2}} .
\end{aligned}
$$

\section{MAIN RESUltS}

The lines $M X, M Y, M Z$ and the corresponding sides $B C, C A, A B$ have the same angle named the Miquel angle determined by the inscribed triangle $X Y Z$, which is a direct measured angle and change between 0 and $\pi$ (Figure 2).

\section{Proposition 4.6.}

$$
\begin{aligned}
& \text { (a) } A M=\frac{b c \sqrt{b^{2}(1-\beta)^{2}-2(1-\beta) \gamma S_{A}+c^{2} \gamma^{2}}}{\sqrt{E^{2}+S^{2}}}=\frac{a R_{a}}{O_{b} O_{c}}, \\
& \text { (b) } B M=\frac{c a \sqrt{c^{2}(1-\gamma)^{2}-2(1-\gamma) \alpha S_{B}+a^{2} \alpha^{2}}}{\sqrt{E^{2}+S^{2}}}=\frac{b R_{b}}{O_{c} O_{a}}, \\
& \text { (c) } C M=\frac{a b \sqrt{a^{2}(1-\alpha)^{2}-2(1-\alpha) \beta S_{C}+b^{2} \beta^{2}}}{\sqrt{E^{2}+S^{2}}}=\frac{c R_{c}}{O_{a} O_{b}} .
\end{aligned}
$$

Proof. We have:

$$
\begin{gathered}
A M^{2}=\left(1-x_{M}\right)^{2} S_{A}+y_{M}^{2} S_{B}+z_{M}^{2} S_{C}=\left(y_{M}+z_{M}\right)^{2} S_{A}+y_{M}^{2} S_{B}+z_{M}^{2} S_{C} \\
=c^{2} y_{M}^{2}+2 y_{M} z_{M} S_{A}+b^{2} z_{M}^{2}=\frac{b^{2} c^{2}}{\left(E^{2}+S^{2}\right)^{2}}\left\{b^{2}\left[\left(a^{2}-\lambda\right)(1-\beta)+c^{2} \gamma\right]^{2}\right.
\end{gathered}
$$




$$
\begin{gathered}
\left.+2\left[\left(a^{2}-\lambda\right)(1-\beta)+c^{2} \gamma\right]\left[b^{2}(1-\beta)+\left(a^{2}-\lambda^{\prime}\right) \gamma\right] S_{A}+c^{2}\left[b^{2}(1-\beta)+\left(a^{2}-\lambda^{\prime}\right) \gamma\right]^{2}\right\} \\
=\frac{b^{2} c^{2}}{\left(E^{2}+S^{2}\right)^{2}}\left\{b^{2}\left[\left(S_{\omega}-\lambda-S_{A}\right)(1-\beta)+c^{2} \gamma\right]^{2}\right. \\
+2\left[\left(S_{\omega}-\lambda-S_{A}\right)(1-\beta)+c^{2} \gamma\right]\left[b^{2}(1-\beta)+\left(S_{\omega}-\lambda^{\prime}-S_{A}\right) \gamma\right] S_{A} \\
\left.+c^{2}\left[b^{2}(1-\beta)+\left(S_{\omega}-\lambda^{\prime}-S_{A}\right) \gamma\right]^{2}\right\} \\
=\frac{b^{2} c^{2}}{\left(E^{2}+S^{2}\right)^{2}}\left\{b^{2}\left[\left(E-S_{A}\right)(1-\beta)+c^{2} \gamma\right]^{2}\right. \\
+2\left[\left(E-S_{A}\right)(1-\beta)+c^{2} \gamma\right]\left[b^{2}(1-\beta)-\left(E+S_{A}\right) \gamma\right] S_{A} \\
\left.+c^{2}\left[b^{2}(1-\beta)-\left(E+S_{A}\right) \gamma\right]^{2}\right\} \\
=\frac{b^{2} c^{2}}{\left(E^{2}+S^{2}\right)^{2}}\left\{b^{2}\left[\left(E-S_{A}\right)^{2}+2\left(E-S_{A}\right) S_{A}+b^{2} c^{2}\right](1-\beta)^{2}\right. \\
\left.+b^{2} c^{2}\left(E-S_{A}\right)-\left(E+S_{A}\right)\left(E-S_{A}\right) S_{A}+b^{2} c^{2} S_{A}-b^{2} c^{2}\left(E+S_{A}\right)\right](1-\beta) \gamma \\
=\frac{\left.\left.b^{2} c^{2}-2\left(E+S_{A}\right) S_{A}+\left(E+S_{A}\right)^{2}\right] \gamma^{2}\right\}=}{\left(E^{2}+S^{2}\right)^{2}}\left\{b^{2}\left(E^{2}+S^{2}\right)(1-\beta)^{2}-2\left(E^{2}+S^{2}\right) S_{A}(1-\beta) \gamma+c^{2}\left(E^{2}+S^{2}\right) \gamma^{2}\right\} \\
=\frac{b^{2} c^{2}}{E^{2}+S^{2}}\left[b^{2}(1-\beta)^{2}-2(1-\beta) \gamma S_{A}+c^{2} \gamma^{2}\right] .
\end{gathered}
$$

From the law of sinus we have $2 \sin \theta=\frac{A M}{R_{a}}=\frac{B M}{R_{b}}=\frac{C M}{R_{c}}$ and from the Proposition $4.1 \frac{A M}{R_{a}}=\frac{a}{O_{b} O_{c}}, \frac{B M}{R_{b}}=\frac{b}{O_{c} O_{a}}, \frac{C M}{R_{c}}=\frac{c}{O_{a} O_{b}}$.

Therefore, the triangles $A B C$ and $O_{a} O_{b} O_{c}$ are similar and its similarity ratio is $2 \sin \theta=$ $\frac{a}{O_{b} O_{c}}=\frac{2 S}{\sqrt{E^{2}+S^{2}}}$, from where $\sin \theta=\frac{S}{\sqrt{E^{2}+S^{2}}}$ (Figure 4).

Proposition 4.7. Between the Miquel and Brocard angles exist the following relation:

$$
S_{\omega}=S_{\theta}+a^{2} \alpha+b^{2} \beta+c^{2} \gamma .
$$

Proof. We have: $\cos ^{2} \theta=1-\sin ^{2} \theta=1-\frac{S^{2}}{E^{2}+S^{2}}=\frac{E^{2}}{E^{2}+S^{2}}$. So $\cos \theta=\frac{S_{\omega}-\lambda}{\sqrt{E^{2}+S^{2}}}$ and $S_{\theta}=S \cot \theta=S \frac{S_{\omega}-\lambda}{S}=S_{\omega}-\left(a^{2} \alpha+b^{2} \beta+c^{2} \gamma\right)$.

Remark 4.1. The Miquel angle is right if and only if the triangle $X Y Z$ is pedal triangle.

\section{ISOTOMIC INSCRIBED TRIANGLES}

If $X^{\prime} Y^{\prime} Z^{\prime}$ and $X Y Z$ are isotomic inscribed triangles then $X^{\prime}=(0, \alpha, 1-\alpha)$, $Y^{\prime}=(1-\beta, 0, \beta), Z^{\prime}=(\gamma, 1-\gamma, 0)$. Consequently all results referred to the triangle $X^{\prime} Y^{\prime} Z^{\prime}$ can be obtained from above results replacing $\alpha, \beta, \gamma$ by $1-\alpha, 1-\beta, 1-\gamma$ respectively. For example

$$
O_{b}^{\prime} O_{c}^{\prime}=\frac{a}{2 S} \sqrt{\left(\lambda^{\prime}-S_{\omega}\right)^{2}+S^{2}}=\frac{a}{2 S} \sqrt{\left(S_{\omega}-\lambda\right)^{2}+S^{2}}=O_{b} O_{c},
$$




$$
\begin{aligned}
& O_{c}^{\prime} O_{a}^{\prime}=\frac{b}{2 S} \sqrt{\left(\lambda^{\prime}-S_{\omega}\right)^{2}+S^{2}}=\frac{b}{2 S} \sqrt{\left(S_{\omega}-\lambda\right)^{2}+S^{2}}=O_{c} O_{a}, \\
& O_{a}^{\prime} O_{b}^{\prime}=\frac{c}{2 S} \sqrt{\left(\lambda^{\prime}-S_{\omega}\right)^{2}+S^{2}}=\frac{c}{2 S} \sqrt{\left(S_{\omega}-\lambda\right)^{2}+S^{2}}=O_{a} O_{b} .
\end{aligned}
$$

It follows that the triangles $O_{a} O_{b} O_{c}$ and $O_{a}^{\prime} O_{b}^{\prime} O_{c}^{\prime}$ are congruent.

Proposition 5.8. If $\theta^{\prime}$ is the Miquel angle of the isotomic inscribed triangle $X^{\prime} Y^{\prime} Z^{\prime}$ then

$$
\begin{aligned}
& \text { (a) } S_{\omega}=-S_{\theta^{\prime}}+a^{2} \alpha+b^{2} \beta+c^{2} \gamma \\
& \text { (b) } \theta+\theta^{\prime}=\pi .
\end{aligned}
$$

Proof. We have:

(a) $S_{\theta^{\prime}}=S_{\omega}-\left[a^{2}(1-\alpha)+b^{2}(1-\beta)+c^{2}(1-\gamma)=-S_{\omega}+a^{2} \alpha+b^{2} \beta+c^{2} \gamma\right.$

(b) $S_{\theta}+S_{\theta}^{\prime}=S \cot \theta+S \cot \theta^{\prime}=0$. Therefore $\frac{\sin \left(\theta+\theta^{\prime}\right)}{\sin \theta \cdot \sin \theta^{\prime}}=0$,

i.e. $\theta+\theta^{\prime}=\pi[5]$.

Remark 5.2. For $\alpha=\beta=\gamma=0$ the Miquel point coincide with the $1^{\text {st }}$ Brocard point $\Omega=\left(c^{2} a^{2}: a^{2} b^{2}: b^{2} c^{2}\right)$ (Figure 1) and for $\alpha=\beta=\gamma=1$ we obtain the $2^{\text {nd }}$ Brocard point $\Omega^{\prime}=\left(a^{2} b^{2}: b^{2} c^{2}: c^{2} a^{2}\right)[6]$.

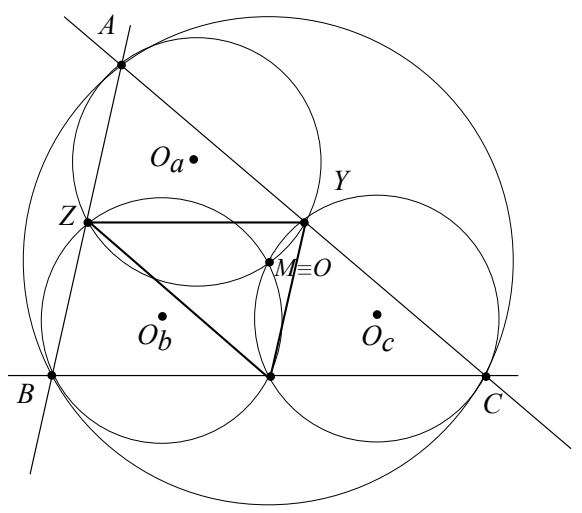

Figure 5 .

If $\alpha=\beta=\gamma=\frac{1}{2}$ the Miquel point coincide with the circumcenter $O=\left(a^{2} S_{A}: b^{2} S_{B}: c^{2} S_{C}\right)$ of the reference triangle $A B C$ (Figure 5) [6].

\section{REFERENCES}

[1] Barycentric coordinate system, https:/ / en.wikipedia.org/wiki/Barycentric coordinate system

[2] Conway triangle notation, https://en.wikipedia.org/wiki/Conway triangle notation

[3] Brocard points, https://en.wikipedia.org/wiki/Brocard points

[4] Miquel's theorem, https://en.wikipedia.org/wiki/Miquel's theorem

[5] Schmidt, E., Circumcenters of Residuals Triangles, Forum Geometricorum, 3 (2003), 207-214

[6] Kimberling, C., Encyclopedia of Triangle Centers, http://faculty.evansville.edu/ck6/encyclopedia/ETC.html 440095 SATU MARE,

AVRAM IANCU STR., NO 58, SC. A, AP. 8

ROMANIA

Email address: d. sandor.kiss@gmail.com 